\title{
Articulatory suppression and the irrelevant- speech effect in short-term memory: Does the locus of suppression matter?
}

\author{
THOMAS C. TOPPINO and ANTHONY PISEGNA \\ Villanova University, Villanova, Pennsylvania
}

\begin{abstract}
Immediate serial recall for letter sequences was impaired when irrelevant speech (IS) was presented throughout stimulus input and a subsequent rehearsal interval. This irrelevant-speech effect was eliminated when participants engaged in articulatory suppression (repeated articulation of one or more digits) during stimulus input but not when suppression occurred during the postinput rehearsal period. Also, changing-state suppression (articulation of multiple items) impaired the overall level of performance more than did steady-state suppression (repetition of a single item), whereas both forms of suppression had the same influence on the IS effect. Our results suggest that the locus of suppression (during or after stimulus input) may have contributed to discrepant findings in the prior literature regarding the influence of articulatory suppression on the IS effect. We consider the implications of our findings for three prominent models of immediate memory: the working memory model, the objectoriented episodic record model, and the feature model.
\end{abstract}

Colle and Welsh (1976) demonstrated that exposure to irrelevant speech (IS) impairs immediate serial recall of visually presented verbal information. This $I S$ effect has been replicated and extended by numerous other researchers (see Neath, 2000, for a review). For example, the IS effect occurs when to-be-remembered information is presented auditorily as well as visually (e.g., Hanley \& Broadbent, 1987); the irrelevant auditory input does not have to involve speech (e.g., Jones \& Macken, 1993); and the IS effect is greater when the irrelevant input consists of changing-state stimulation involving the presentation of multiple items, as opposed to steady-state stimulation involving the continual repetition of a single item (e.g., Jones, Madden, \& Miles, 1992).

Immediate serial recall is also impaired when participants articulate an irrelevant word or phrase during the memory task (the articulatory suppression effect; Salamé $\&$ Baddeley, 1982). The deleterious effect of suppression usually is greater than that of IS (e.g., Salamé \& Baddeley, 1982) and is greater when participants pronounce multiple irrelevant items (changing-state suppression), as opposed to repeatedly articulating a single irrelevant item (steady-state suppression; e.g., Macken \& Jones, 1995).

An important issue concerns whether and how suppression reduces the IS effect. However, results have been equivocal when visually presented stimuli are in-

We thank Ronak Chokshi for his assistance in collecting and analyzing the data. We also appreciate the helpful comments of Jim Nairne, Ian Neath, Mike Page, and two anonymous reviewers. Correspondence concerning this article should be addressed to T. C. Toppino, Department of Psychology, Villanova University, 800 Lancaster Ave., Villanova, PA 19085 (e-mail: thomas.toppino@villanova.edu). volved. Salamé and Baddeley (1982) presented lists visually and found that the IS effect was abolished by steady-state suppression (repetition of one word). That is, under conditions of suppression, the presence or absence of IS did not influence immediate recall. This finding was replicated in several other studies (Hanley, 1997; Miles, Jones, \& Madden, 1991; Salamé \& Baddeley, 1987). However, Macken and Jones (1995, Experiment 5) found that the IS effect was not attenuated by steady-state suppression and was not eliminated even by changing-state suppression (see also Norris, Baddeley, \& Page, 2004).

The previously cited experiments differ in two potentially critical ways. Macken and Jones (1995) required suppression only during a postinput retention interval or "rehearsal phase," in which participants articulated silently. In contrast, Hanley (1997) and Salamé and Baddeley $(1982,1987)$ required suppression during the input phase of each trial, in which the irrelevant articulation was vocalized. Miles et al. (1991) reported that vocalized, steady-state suppression eliminated the IS effect regardless of when suppression occurred.

Macken and Jones (1995, Experiments 1 and 2) found that vocalized suppression impaired performance more than did silently mouthed suppression. They proposed that the conflicting findings could be reconciled if the more potent vocalized form of suppression were assumed to abolish the IS effect, regardless of type and locus of suppression. This conclusion rests strongly on the fact that Miles et al. (1991, Experiments 2 and 3) failed to find an IS effect when participants engaged in vocalized, steady-state suppression, and that they obtained no significant interaction to indicate that the results depended on the locus of suppression. Upon closer 
examination, however, the data of Miles et al. do not seem convincing.

In Miles et al.'s (1991) Experiment 2, an IS effect was not obtained in any condition, including the control condition. This renders their failure to obtain an IS effect under conditions of suppression uninterpretable. In their Experiment 3, an IS effect occurred without suppression but not with suppression. Although the interaction with the locus of suppression was not significant, inspection of the data suggests that the IS effect may have been eliminated primarily when suppression occurred during stimulus input. In the latter condition, the IS condition produced only $3.9 \%$ more errors than did the no-speech condition. However, when suppression occurred after input, the speech condition yielded a substantial $27.4 \%$ more errors than did the no-speech condition. Thus, it seems possible that the effect of suppression on the IS effect does depend on the locus of suppression and that the effect simply went undetected by Miles et al.

The present experiment was designed to assess whether the locus of vocalized suppression can be shown to influence the IS effect when stimuli are presented visually. Our intent was not to parametrically explore the procedural variations represented in previous studies, but simply to hold the locus of IS constant while systematically varying the locus of suppression. We used changing-state IS and held its locus constant such that speech was presented continuously during both stimulus input and a postinput retention (rehearsal) interval, or it was kept absent from both. Our primary concern was how the IS effect (defined as the difference in performance between the speech and no-speech conditions) might be affected by the locus of vocalized suppression: no suppression versus suppression during stimulus input only versus suppression during the retention (rehearsal) interval only. We also varied the use of steady-state or changing-state suppression. An obtained effect of the locus of suppression would suggest that the locus of suppression may have contributed to the discrepant findings reviewed above. In addition, the results may be of theoretical interest because the major theories of immediate memory differ regarding the relationship between suppression and IS.

According to Baddeley (1986, 2000b), working memory involves a central executive and several subsidiary systems. The IS and suppression effects are explained in terms of the phonological loop subsystem, which consists of a phonological short-term store and an articulatory rehearsal loop. Articulation functions to refresh stored information (rehearsal) and to recode visual information for entry into the phonological store. Presumably, IS impairs performance by interfering with information in the phonological store, whereas suppression impedes rehearsal by occupying the rehearsal loop. Both steady-state and changing-state suppression during visual stimulus input should occupy the rehearsal loop and eliminate the IS effect by preventing stimuli from entering the phono- logical store. However, suppression following input occurs too late to prevent stimuli from being recoded and, thus, should not eliminate the IS effect.

According to the object-oriented episodic record (OOER) model (Jones, 1993; Macken \& Jones, 1995), stimuli are encoded as amodal, abstract objects; serial order is encoded by episodic pointers linking successive objects; and items with common origins are organized into sequences or streams. Presumably, the IS and suppression effects are produced by essentially the same underlying mechanism. That is, pointers (order cues) in the stream of to-be-remembered items undergo interference from pointers in streams generated by IS or suppression. Greater interference should be obtained with changingstate than with steady-state speech or articulation because the former presumably are represented as streams of multiple objects connected by pointers, whereas the latter are represented as single items with self-referential links that produce relatively little impairment (Macken \& Jones, 1995). Both IS and suppression can interfere with order cues regardless of their locus (during or following input), and their effects may be redundant. Suppression should abolish the IS effect to the extent that interference from suppression alone is so great that IS can have little additional effect on performance. For similar reasons, the effect of changing- versus steady-state suppression should be attenuated in conditions involving changing-state IS (Macken \& Jones, 1995).

Finally, according to Neath's (2000) adaptation of Nairne's (1990) feature model, a stimulus is represented as a set of features, and both IS and suppression are assumed to interfere with to-be-remembered items through the common mechanism of "feature adoption." That is, features of stimuli in primary memory are lost because they are replaced by features of the IS and/or articulated material. However, suppression is expected to be more disruptive of performance because it requires more attentional resources. As in the O-OER model, suppression and IS can interfere regardless of whether they occur during or after input, and suppression should abolish the IS effect if suppression alone produces nearly maximal interference.

\section{METHOD}

\section{Participants and Design}

The participants were 108 introductory psychology students, 54 of whom were randomly assigned to each of two groups generated by a 2 (suppression type: steady- vs. changing-state) $\times 3$ (suppression locus: none vs. input vs. rehearsal) $\times 2$ (speech: irrelevant speech vs. quiet) $\times 7$ (serial position) mixed factorial design with the first factor manipulated between participants.

\section{Materials and Procedure}

On each of 108 trials, the participants viewed a seven-consonant list. Each consonant was centrally presented on a computer screen for $800 \mathrm{msec}$. A 200-msec blank screen followed. Each list consisted of a random sequence of the consonants $\mathrm{C}, \mathrm{F}, \mathrm{H}, \mathrm{K}, \mathrm{M}, \mathrm{Q}$, and $\mathrm{R}$.

Each trial consisted of a 7-sec input phase, a 7-sec "rehearsal" phase, and a serial recall phase. To start, the word "ready" appeared 
for $1 \mathrm{sec}$. Then, the list was presented at a rate of one item per second. After the last consonant, an asterisk appeared for $1 \mathrm{sec}$, followed by the word "wait" for $7 \mathrm{sec}$, and then the words "begin recall." The period between the ready signal and the asterisk was defined as the input phase, whereas the interval between the asterisk and the recall signal was defined as the rehearsal phase. The participants recalled using a response sheet containing seven blank spaces for each trial. They were instructed to write from left to right without backtracking and to guess when uncertain.

On IS trials, the participants heard a Portuguese literary passage presented in a female voice via a cassette tape player at an average intensity of $70 \mathrm{~dB}$. (No participant spoke or comprehended Portuguese.) The speech was presented continuously throughout the input and rehearsal phases of the trial, and the participants were instructed to ignore it.

On a suppression trial, the participants engaged in irrelevant articulation during a portion of the trial. Depending on the locus of suppression, the participants articulated from the "ready" signal to the asterisk or from the asterisk to the "begin recall" signal. Changingstate suppression involved repeating aloud the digits " 1 " through "7" (e.g., 1, 2, 3, etc.). Steady-state suppression involved repeating a single number (e.g., 3, 3, 3, etc.) selected randomly from the set 1 through 7 . Vocalizations were paced at a rate of two digits per second.

The speech $\times$ suppression conditions were presented in six 18trial blocks representing each of the six combinations of two levels of speech and three loci of suppression. Prior to each block, the participants were told which suppression condition would follow. The participants took a 30 -sec rest between blocks. The presentation order of blocks was counterbalanced using a Latin-square principle such that each condition appeared once in each position and was never preceded or succeeded by the same condition more than once. Each block order was administered to an equal number of participants in each group.

\section{RESULTS}

A consonant was considered correct only if it was recalled in the correct serial position. Preliminary analyses indicated that serial position did not affect any theoretical or empirical conclusions of interest. Therefore, the data were collapsed across serial position and were submitted to a 2 (type of suppression) $\times 3$ (locus of suppression) $\times 2$ (speech condition) mixed analysis of variance with repeated measures on the last two factors. The significance level was set at $p=.05$ for all analyses.

Figure 1 presents the mean percentage of errors. There was a significant effect of the locus of suppression $\left[F(2,212)=272.19, M S_{\mathrm{e}}=152.05\right]$. Tukey HSD post hoc comparisons indicated that the percentage of errors was lowest with no suppression, was significantly higher with suppression during rehearsal, and was highest with suppression during input. There was also a significant main effect of IS $\left[F(1,106)=28.46, M S_{\mathrm{e}}=78.86\right]$, which was qualified by an IS $\times$ locus of suppression interaction $\left[F(2,212)=5.66, M S_{\mathrm{e}}=76.90\right]$. To probe this interaction, the simple main effect of IS (speech vs. no speech) was analyzed separately for each locus-of-suppression condition. There was an IS effect (i.e., more errors occurred in the presence of IS than without speech) in the no-suppression and suppression-at-rehearsal conditions, but not in the suppression-at-input condition $[F \mathrm{~s}(1,318)=$ $28.59,11.18$, and 0.39 , respectively, $\left.M S_{\mathrm{e}}=77.56\right]$.
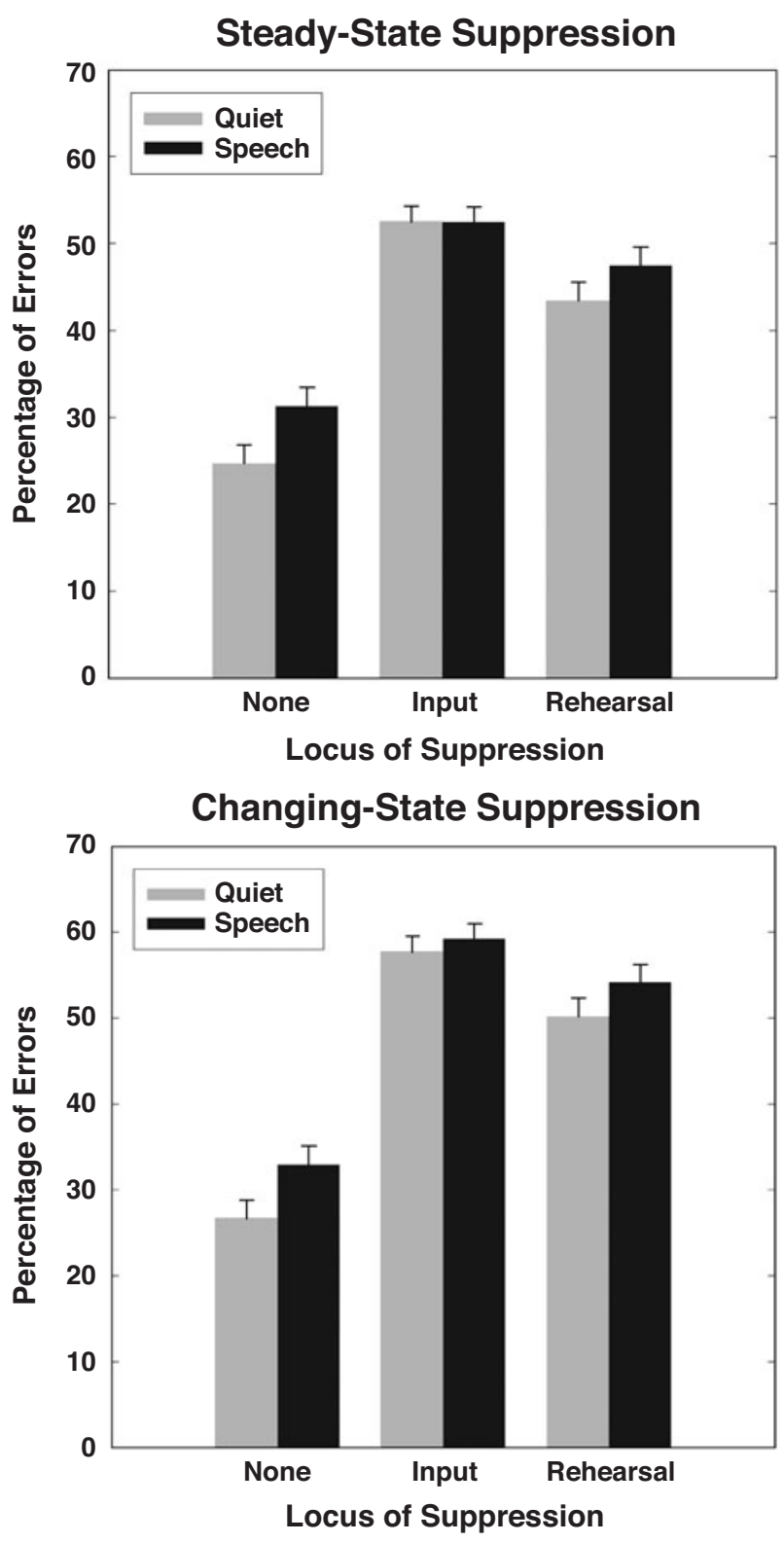

Figure 1. Mean percentage of errors in serial recall (with standard error bars) as a function of the locus of suppression and whether irrelevant speech was presented (speech) or not (quiet). The top and bottom panels show the mean errors for groups that received steady-state and changing-state suppression, respectively.

Type of suppression significantly affected performance $\left[F(1,106)=4.67, M S_{\mathrm{e}}=5,717.03\right]$, with more recall errors for changing-state than for steady-state suppression. The locus $\times$ type of suppression interaction was marginally significant $\left[F(2,106)=2.46, M S_{\mathrm{e}}=152.05, p<\right.$ $.10]$, reflecting the unsurprising fact that the difference between type-of-suppression groups was minimal in blocks not requiring suppression. The effect of type of suppression did not vary with the presence or absence of IS $\left[F(1,106)<1.00, M S_{\mathrm{e}}=78.86\right]$. 


\section{DISCUSSION}

We varied the locus of suppression and found that suppression during stimulus input impaired overall performance more than did suppression following input. Although a previous study by Miles et al. (1991) did not report such an asymmetry of locus, power was comparatively low (a between-participants variable with 12 participants per group vs. a within-participants variable with 108 participants in the present research). ${ }^{1}$ Miles et al. also confounded the locus of suppression with its duration. Suppression was $2 \sec (25 \%)$ longer during rehearsal than during input, potentially compensating somewhat for the less disruptive effect of suppression during rehearsal. Finally, the observed asymmetry is consistent with the working-memory and O-OER models (e.g., Baddeley, 2000b; Macken \& Jones, 1995) as will be discussed below, but it is unclear whether asymmetry would be expected according to the feature model (e.g., Neath, 2000).

We found that, when IS was presented both during and following stimulus presentation, suppression during input abolished the IS effect, whereas postinput suppression had no significant influence on the IS effect. Our design does not allow us to determine whether postinput suppression simply occurred too late to alter the IS effect and whether it might have attenuated the effect of a more limited IS manipulation (e.g., IS following input only). Nevertheless, our results indicate that the locus of suppression does matter, and the findings are consistent with the hypothesis that the locus of suppression may have contributed to discrepancies in the prior literature.

Whenever suppression has occurred during visual stimulus input, the IS effect has been eliminated (Hanley, 1997; Miles et al., 1991; Salamé \& Baddeley, 1982, 1987). However, suppression following input typically has failed to eliminate the IS effect. Both we and Macken and Jones (1995) found that steady-state suppression following input failed to reduce the IS effect. A similar, but nonsignificant, pattern of results was obtained by Miles et al. (1991, Experiment 3). Finally, neither we nor Macken and Jones (1995) eliminated the IS effect with postinput changing-state suppression, although the latter investigators did find a greater reduction with changing- than with steady-state suppression. The above generalization is also consistent with very recent findings obtained by Norris et al. (2004).

Our results are consistent with strong predictions derived from the phonological loop hypothesis of Baddeley's $(1986,2000 b)$ working memory model. Articulatory suppression during input should abolish the IS effect by preventing visual stimuli from being recoded and entered into the phonological store where they can suffer interference from IS. Because suppression following input does not prevent visual stimuli from being encoded into the phonological store, it may impair performance by preventing rehearsal, but it cannot prevent the IS effect.

The working memory model also explains why suppression impaired overall performance more when it oc- curred during, rather than after, input. In the former case, items that were denied access to the phonological store were presumably processed by another component of the system (e.g., the episodic buffer [Baddeley, 2000a]) that may be less efficient for encoding and storing sequential verbal information.

In contrast to the working memory model, the O-OER model (Jones, 1993) did not predict our results. However, a post hoc account seemed possible. Because suppression impaired overall performance more when it occurred during input as opposed to after input, one might argue that interference from suppression was great enough to eliminate the IS effect in the former, but not in the latter, case. The O-OER model seems to account for better overall performance when suppression follows input because, in that condition, items can be rehearsed during presentation, thereby strengthening the episodic pointers that encode order information. However, suppression during input may impede rehearsal, resulting in weaker episodic pointers and poorer performance. Despite its ability to account for the asymmetrical suppression effects, however, the O-OER model does not seem to provide a satisfactory account of how suppression influenced the IS effect.

According to the O-OER model, suppression should eliminate the IS effect to the extent that suppression alone generates maximal interference with order cues so that IS can produce no further disruption. However, suppression at input yielded means of $52.5 \%$ and $57.8 \%$ errors for steady-state and changing-state suppression, respectively, neither of which approaches a ceiling or floor effect. Thus, in this task, which depended very heavily on memory for order information (i.e., each trial was simply a different random permutation of the same seven items), participants seemed to have a substantial amount of order information available in spite of suppression at input. It is, therefore, unclear why changing-state IS was unable to produce additional interference. ${ }^{2} \mathrm{~A}$ further indication that the IS effect was abolished in the absence of maximal interference is found in the fact that changing-state suppression at input interfered with serial recall more than did steady-state suppression at input. Because the O-OER model attributes this difference to greater interference with order information in the former condition, steady-state suppression at input must not have produced maximal interference and, according to the model, should not have eliminated the IS effect.

The feature model, like the O-OER model, attributes the effects of both suppression and IS to interference that can occur during or following input. However, the mechanism of interference is different for the feature model (interference with feature information) and the O-OER model (interference with order information) so that predictions may differ (Jones \& Tremblay, 2000). An advantage of the feature model is that it is stated with sufficient precision that the plausibility of its explanations can be verified by simulations. Neath (2000) reported a simulation indicating that steady-state suppression during visual 
input (and recall) eliminated the IS effect, which is consistent with our results. However, suppression during the retention interval has not been implemented in the feature model, so it remains to be determined whether the model can account for the full pattern of our results.

Our findings revealed a changing-state effect (poorer overall performance with changing-state than with steady-state suppression) but no difference in the degree to which the two types of suppression influenced the IS effect. The changing-state effect is the signature prediction of the O-OER model (e.g., Jones et al., 1992), which attributes both it and the influence of suppression on the IS effect to interference with order cues in the target stream of objects. However, in applying this hypothesis to our data, it is unclear how differences in interference associated with changing- and steady-state suppression, respectively, can account for the overall changing-state effect without also impacting differently on the IS effect.

Although an account of the changing-state effect was a late addition to the working memory model, the effect can be explained by assuming that changing-state suppression places greater demands on the limited resources of the central executive (e.g., Larsen \& Baddeley, 2003). The model, however, is compatible with our findings because the changing-state effect and the influence of suppression on the IS effect are attributed to unrelated mechanisms. The former is attributed to resource demands, and the latter presumably occurs because both types of suppression occupy the rehearsal loop and prevent recoding of visually presented information.

The feature model also attributes the overall changingstate effect and the influence of suppression on the IS effect to different mechanisms. The former can be explained in terms of greater attentional demands, ${ }^{3}$ whereas the latter is attributed to interference. Unfortunately, these conceptually independent mechanisms have a multiplicative relationship when implemented. Thus, although the relevant simulations have not been conducted, the feature model seems to predict that, if changing- and steady-state suppression have different effects on overall performance, they also should have different degrees of influence on the IS effect.

In summary, the locus of suppression may have contributed to discrepancies in the immediate-memory literature. Generally, suppression during input has abolished the IS effect, whereas suppression following input has not. Prominent theories of immediate memory differ in how easily they can accommodate these findings. The O-OER model seems to require modification or elaboration, because currently, it neither predicts our results nor provides a convincing post hoc explanation. The feature model accounts for our results with suppression at input, but, at present, does not address suppression effects during rehearsal. In contrast, our findings conform very well to strong predictions from Baddeley's $(1986,2000 \mathrm{~b})$ working memory model. It is noteworthy, however, that the literature on immediate memory is a thicket of empirical and theoretical issues about which there is con- siderable contention. Thus, although Baddeley's (2000b) working memory model fares well with the locus of suppression, the model has been criticized for, among other things, inadequately explaining the IS effect and inadequately accounting for similarities in the effects of suppression and IS (e.g., Macken \& Jones, 2003; Neath, Farley, \& Surprenant, 2003; but see also Baddeley \& Larsen, 2003; Larsen \& Baddeley, 2003; Page \& Norris, 2003). Clearly, much additional research is in order.

\section{REFERENCES}

BAdDeley, A. [D.] (1986). Working memory. New York: Oxford University Press.

BADDELEY, A. [D.]. (2000a). The episodic buffer: A new component of working memory? Trends in Cognitive Sciences, 4, 417-423.

BADDELEY, A. [D.] (2000b). The phonological loop and the irrelevant speech effect: Some comments on Neath (2000). Psychonomic Bulletin \& Review, 7, 544-549.

BADDELEY, A. [D.], \& LARSEN, J. D. (2003). The disruption of STM: A response to our commentators. Quarterly Journal of Experimental Psychology, 56A, 1301-1306.

Colle, H. A., \& Welsh, A. (1976). Acoustic masking in primary memory. Journal of Verbal Learning \& Verbal Behavior, 15, 17-32.

Hanley, J. R. (1997). Does articulatory suppression remove the irrelevant speech effect? Memory, 5, 423-431.

Hanley, J. R., \& BroadBent, C. (1987). The effect of unattended speech on serial recall following auditory presentation. British Journal of Psychology, 78, 287-297.

JoNES, D. M. (1993). Objects, streams, and threads of auditory attention. In A. [D.] Baddeley \& L. Weiskrantz (Eds.), Attention: Selection, awareness, and control. A tribute to Donald Broadbent (pp. 87-104). Oxford: Oxford University Press, Clarendon Press.

Jones, D. M., \& MACKEN, W. J. (1993). Irrelevant tones produce an irrelevant speech effect: Implications for phonological coding in working memory. Journal of Experimental Psychology: Learning, Memory, \& Cognition, 19, 369-381.

Jones, D. M., Madden, C., \& Miles, C. (1992). Privileged access by irrelevant speech to short-term memory: The role of changing state. Quarterly Journal of Experimental Psychology, 44A, 645-669.

Jones, D. M., \& Tremblay, S. (2000). Interference in memory by process or content? A reply to Neath (2000). Psychonomic Bulletin \& Review, 7, 550-558.

LARSEN, J. D., \& BADDELEY, A. [D.] (2003). Disruption of verbal STM by irrelevant speech, articulatory suppression, and manual tapping: Do they have a common source? Quarterly Journal of Experimental Psychology, 56A, 1249-1268.

Macken, W. J., \& Jones, D. M. (1995). Functional characteristics of the inner voice and the inner ear: Single or double agency? Journal of Experimental Psychology: Learning, Memory, \& Cognition, 21, 436-448.

MACKEN, W. J., \& Jones, D. M. (2003). Reification of phonological storage. Quarterly Journal of Experimental Psychology, 56A, 12791288.

Miles, C., Jones, D. M., \& Madden, C. A. (1991). Locus of the irrelevant speech effect in short-term memory. Journal of Experimental Psychology: Learning, Memory, \& Cognition, 17, 578-584.

NAIRNE, J. S. (1990). A feature model of immediate memory. Memory \& Cognition, 18, 251-269.

NEATH, I. (2000). Modeling the effects of irrelevant speech on memory. Psychonomic Bulletin \& Review, 7, 403-423.

NeATH, I., Farley, L. A., \& Surprenant, A. M. (2003). Directly assessing the relationship between irrelevant speech and articulatory suppression. Quarterly Journal of Experimental Psychology, 56A, 1269-1278.

Norris, D., BAdDeley, A. [D.], \& PAGe, M. P. A. (2004). Retroactive effects of irrelevant speech on serial recall from short-term memory. Journal of Experimental Psychology: Learning, Memory, \& Cognition, 30, 1093-1105 
Page, M. P. A., \& Norris, D. G. (2003). The irrelevant sound effect: What needs modeling, and a tentative model. Quarterly Journal of Experimental Psychology, 56A, 1289-1300.

Salamé, P., \& Baddeley, A. [D.] (1982). Disruption of short-term memory by unattended speech: Implications for the structure of working memory. Journal of Verbal Learning \& Verbal Behavior, 21, 150164.

Salamé, P., \& BAdDEley, A. [D.] (1987). Noise, unattended speech and short-term memory. Ergonomics, 30, 1185-1194.

\section{NOTES}

1. Comparisons of articulatory suppression during input versus rehearsal in Miles et al.'s (1991) Experiment 3 were based on 240 total observations per experimental condition, whereas the relevant comparisons in the present experiment involved 1,944 observations per condition.

2. An alternative approach within the context of the O-OER model is to assume that maximal interference never completely destroys order cues so that maximal interference will still allow moderate levels of performance. Given this assumption, one might argue that the greater degree of interference produced by suppression at input in our experiment was maximal and sufficient to abolish the IS effect, whereas less interference produced by suppression at rehearsal was not. However, this approach does not readily explain why the IS effect was eliminated by steady-state suppression at input but not by changing-state suppression during rehearsal even though the two conditions produced approximately the same percentage of errors $[52.5 \%$ vs. $52.2 \%$, respectively; $t(106)=.82]$. A more general problem with the approach is that, without an independent method of determining the level of interference, the interference from suppression can be deemed to be maximal or not depending on whether suppression does or does not eliminate the IS effect. Unfortunately, this would leave us with a circular explanation and an untestable theoretical formulation.

3. Although Neath (2000) did not model the changing-state effect with articulatory suppression, he did model the changing-state effect with irrelevant speech by assigning to the changing-state condition a relatively lower value of a parameter assumed to reflect the availability of attentional resources. It seems straightforward to generate a parallel account of the changing-state effect with articulatory suppression.

(Manuscript received September 30, 2002; revision accepted for publication March 3, 2004.) 